Military Technical College Kobry El-Kobba

Cairo, Egypt

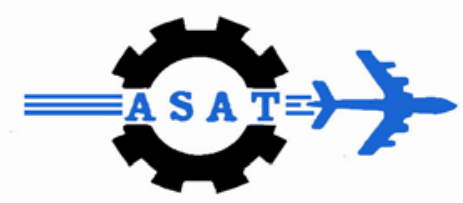

12-th International Conference on Aerospace Sciences \& Aviation Technology

\title{
OPTIMUM RECEIVER UNDER CLASS-A IMPULSIVE NOISE FOR FLAT FADING CHANNELS
}

\author{
EL-Mahdy A. E. , and EL shawadfy S. A.*
}

\begin{abstract}
The paper presents an optimum receiver for digitally modulated signals in additive class-A impulsive noise for flat fading channels. The receiver is based on maximum likelihood (ML) approach. Two algorithms are presented to estimate the unknown fading channel. The convergence properties of the estimation algorithms are investigated and compared. The performance of the receiver is evaluated by simulation. The performance is measured by the bit error probability. Computer simulations are illustrated to validate the theoretical developments. It is shown that the performance of the developed receiver is sensitive to the impulsive index of the noise. Decreasing the value of the impulsive index degrades the performance of the receiver and reduces the receiver resistivity against frequency and time offset.
\end{abstract}

KEY WORDS: class-A impulsive noise, optimum reception, flat fading. 


\section{INTRODUCTION}

In most of digital communication systems, the additive white Gaussian noise (AWGN) channel model is widely applied. However, the assumption of white Gaussian noise alone is sometimes not true. In several situations, the noise accompanied with the signal is non-Gaussian [1]. For example, the communication channels usually exhibit impulsive characteristics, i.e., long bursts due to noise impulses of large amplitude relative to the root mean square noise [2]. Also, the noise generated by a variety of natural and manmade electromagnetic sources exhibits impulsive characteristics [2], [3]. Non-Gaussian impulsive noise is known to be one of the major sources of errors in digital transmission systems. Therefore, a more realistic noise model might be an additive mixture of the Gaussian thermal noise and a non-Gaussian impulsive noise. One of the models that has been proposed to meet these requirements, is the general model derived by Middleton [5]. According to the relation between the durations of the noise impulses and the spectral bandwidth of the receiver, Middleton derived three general classes of the impulsive noise: class-A, B, and C [4], [5]. Class-A model represents the interference arising from sources whose emission spectra are equal to or narrower than the receiver bandwidth. Class-B model represents the interference arising from sources whose emission spectra are broader than the pass-band of the receiver while class- $C$ interference is composed of the sum of class-A and class-B components. In this paper, we use class-A impulsive noise model because it is known to fit closely a variety of nonGaussian noises and also it is analytically tractable model of Gaussian/non-Gaussian noises.

Fading is used to describe the rapid fluctuation of the amplitude of a radio signal over a period of time. Fading is caused by interference between two or more versions of the transmitted signal which arrive at the receiver at slightly different times. These multi-path waves, combine at the receiver antenna to give a resultant signal which can vary widely in amplitude and phase. Multi-path in the radio channel are classified to: (1) changes in signal strength over a time interval, (2) random frequency modulation due to varying Doppler shifts on different multi-path signals, and (3) time dispersion (echoes) caused by multi-path propagation delays.

In this paper, The unknown fading channel is estimated using two different algorithms. The first algorithm estimates the channel for each hypothesis and the second one uses transmitted pilot symbols and uses them to estimate the channel. The performance of the receiver using the two algorithms are performed by computer simulations. The performance of the receiver is derived theoretically and validated by simulations for a QPSK signal as a case study. The paper is organized as follows. In section II, the classA impulsive noise model is briefly reviewed. In section III, the optimal receiver structure for class-A impulsive noise over flat fading channel is derived. In section IV, the simulation experiments are presented to validate the theoretical performance of the receiver. Finally, conclusions are presented in section V. The performance of the designed receiver is evaluated in terms of probability of error. The sensitivity of the receiver to time and frequency offsets is also studied. 


\section{CHARACTERISTICS OF CLASS-A IMPULSIVE NOISE}

Class-A impulsive noise model is a generalized model of the Gaussian noise combined with a non-Gaussian impulsive noise. In this model, a frequency component of the impulsive noise is constrained within the bandwidth of the receiver. The class-A impulsive noise for real channel has a density function $p(n)$, given by [ 6,pp.86 ]:

$$
p(n)=\sum_{m=0}^{\infty} \frac{e^{-A} A^{m}}{\sqrt{2 \pi \sigma_{m}^{2}}} \exp \left(-\frac{n^{2}}{2 \sigma_{m}^{2}}\right)
$$

where the parameter $A$ is called the impulsive index: it is the product of the received average number of impulses per unit time and the duration of an impulse. This parameter defines the impulsiveness of the noise. For small $A$, the noise becomes more impulsive, that is $p(n)$ exhibits large impulsive "tails" and for large $A$, the statistical characteristics of the class-A impulsive noise approach those of Gaussian noise. The probability density function of the class-A impulsive noise for complex channels is given by:

$$
p(n)=\sum_{m=0}^{\infty} \frac{e^{-A} A^{m}}{m ! 2 \pi \sigma_{m}^{2}} \exp \left(-\frac{|n|^{2}}{2 \sigma_{m}^{2}}\right)
$$

The variances $\sigma_{m}^{2}$ are related to the physical parameters and are given by:

$$
\sigma_{m}^{2}=\sigma_{n}^{2} \frac{(m / A)+\Gamma}{1+\Gamma} ; \quad m=0,1,2, \cdots
$$

where the parameter $\sigma_{n}^{2}$ defines the mean variance of the class-A impulsive noise. The model of white class-A combines the presence of an additive man-made noise component with variance $\sigma_{i}^{2}$ and a white Gaussian noise component with variance $\sigma_{G}^{2}$. The parameter $\Gamma$ in (3) is the ratio of the mean power of the Gaussian noise component to the non-Gaussian impulsive noise component. The white Gaussian noise component is presented in class-A noise model to describe the influence of thermal noise which is naturally present in the real physical receiver. Note that $p(n)$ consists of an infinite weighted sum of zero mean Gaussian densities with decreasing weights and increasing variances. An approximation to the model in (1) and (2) can be obtained by limiting the sum to the first three terms only which are found to be sufficient to give excellent approximation to the noise probability density function [7]. In fact, the series should be summed until the factor $A^{m} / m ! \sqrt{2 \pi \sigma_{m}^{2}}$ is smaller than the tolerable error. This factor 
makes the terms decrease quickly as $m$ increases. It is found that three terms are enough for many applications.

Hence, the probability density function of the class-A impulsive noise for complex channels (given by (2)) can be written as:

$$
p(n)=\sum_{m=0}^{2} \frac{e^{-A} A^{m}}{m ! 2 \pi \sigma_{m}^{2}} \exp \left(-\frac{|n|^{2}}{2 \sigma_{m}^{2}}\right)
$$

Another approximation to the model in (1) and (2) can be obtained by the maximum value of its first three terms only. This approximation is valid under the condition that the impulsive index $A$ is sufficiently small [8]. According to this approximation, the probability density function of the class-A impulsive noise for complex channels (given by (2)) can be written as:

$$
p(n)=\max _{m=0,1,2}\left\{\frac{e^{-A} A^{m}}{m ! 2 \pi \sigma_{m}^{2}} \exp \left(-\frac{|n|^{2}}{2 \sigma_{m}^{2}}\right)\right\}
$$

This approximation is used in this thesis to model the generated class-A noise.

\section{DERIVATION OF THE OPTIMUM RECEIVER}

In this section, the optimum receiver over flat fading channel is derived. The channel is assumed to be time invariant with no intersymbol interference (ISI). The fading channel parameter $\alpha$ in this case is given by:

$$
\alpha=q e^{j \theta}
$$

where $q$ denotes the amplitude which is a random variable with Rayleigh distribution:

$$
p(q)=\frac{2 q}{\Omega} \exp \left\{-\frac{q^{2}}{\Omega}\right\} ; \quad q, \Omega>0
$$

where $\Omega=E\left\{q^{2}\right\}$ is the mean square value of $q$. The parameter $\theta$ in (6) denotes the phase which is uniformly distributed from $(-\pi: \pi)$ :

$$
p(\theta)=\frac{1}{2 \pi} ; \quad-\pi<\theta<\pi
$$


Now, the optimum ML receiver to detect one signal out of $M$ is derived. The channel is assumed to be unknown. The received signal over flat fading channel can be written as:

$$
r(t)=\alpha s_{i}(t)+n(t) ; \quad i=1,2, \ldots, M
$$

where $s_{i}(t)$ is the $i^{\text {th }}$ transmitted signal. Writing the waveform in (9) in vector forms yields:

$$
\mathbf{r}=\alpha \mathbf{s}_{i}+\mathbf{n}
$$

where $\mathbf{r}=(r(1), r(2), \ldots, r(K)), \mathbf{s}_{i}=\left(s_{i}(1), s_{i}(2), \ldots, s_{i}(K)\right)$, and $\mathbf{n}=(n(1), n(2), \ldots, n(K))$. Note that $K$ is the number of samples per symbol. The probability density function of the received signal given the transmitting signal $\mathbf{s}_{i}$ and the unknown fading parameter $\alpha$ can be expressed as:

$$
\left.p\left(\mathbf{r} / \mathbf{s}_{i}, \alpha\right)=\sum_{m=0 m ! \sqrt{2 \pi \sigma_{m}^{2}}}^{\infty} \frac{e^{-A} A^{m}}{2 \sigma_{m}^{2}}\left\|\mathbf{r}-\alpha \mathbf{s}_{i}\right\|^{2}\right\}
$$

The $M L$ receiver to detect one out of $M$ signals is that one that maximizes (11) with respect to $i$ but we have unknown parameter $\alpha$ in (11). To handle the incomplete knowledge in (11), the unknown parameter $\alpha$ might be considered as a complex random variable with pdf $p(\alpha)$ and the average likelihood $\int p\left(\mathbf{r} / \mathbf{s}_{i}, \alpha\right) p(\alpha) d \alpha$ might be maximized. To avoid the dependence of the resulting receiver structure upon $p(\alpha)$, the most adverse $p(\alpha)$ might be selected according to the mini-max strategy [9 ].

A more convenient approach is to use the generalized maximum likelihood (GML) rule. In this rule, $\alpha$ is modeled as an unknown parameter, rather than as a random variable yielding an $\mathrm{M}$-ary composites hypothesis testing problem (provided that all the transmitted signal have non-zero energy). The hypothesis $H_{i}$ is then selected for which the generalized maximum likelihood $p\left(\mathbf{r} / \mathbf{s}_{i}, \hat{\alpha}\right)$ is maximum, where $\hat{\alpha}$ is the ML estimate of $\alpha$. Based on (11), the GML rule is implemented by maximizing $p\left(\mathbf{r} / \mathbf{s}_{i}, \alpha\right)$ with respect to $\alpha$ and the index $i$. Maximizing $p\left(\mathbf{r} / \mathbf{s}_{i}, \alpha\right)$ is equivalent to minimize the quantity $\left\|\mathbf{r}-\alpha \mathbf{s}_{i}\right\|$. According to the GML rule, $\min _{\alpha}\left\|\mathbf{r}-\alpha \mathbf{s}_{i}\right\|^{2}=\left\|\mathbf{r}-\hat{\alpha} \mathbf{s}_{i}\right\|^{2}$, and therefore the GML rule is still the minimum distance rule, but the distances are between the received vector and the estimated signal vector $\hat{\alpha} \mathbf{s}_{i}, \quad i=1,2, \ldots, M$. It is clear that the minimum of $\left\|\mathbf{r}-\alpha \mathbf{s}_{i}\right\|^{2}$ with respect to $\alpha$ results in [9]: 


$$
\hat{\alpha}_{i}=\frac{\mathbf{r} \cdot \mathbf{s}_{i}}{\left\|\mathbf{s}_{i}\right\|^{2}}
$$

Note that $\hat{\alpha}_{i}$ is obtained for each hypothesis $i=1,2, \ldots, M$ so, by substituting of (12) into (11), we have $p\left(\mathbf{r} / \mathbf{s}_{i}, \hat{\alpha}_{i}\right)$ and the optimum receiver is obtained by maximizing $p\left(\mathbf{r} / \mathbf{s}_{i}, \hat{\alpha}_{i}\right)$ over the index $i$. Using the approximation given by (5) we maximize

$$
\Delta_{i}=\max _{m=0,1,2}\left\{\ln \left(\frac{e^{-A} A^{m}}{m ! \sqrt{2 \pi \sigma_{m}^{2}}}\right)-\frac{1}{2 \sigma_{m}^{2}}\left\|\mathbf{r}-\hat{\alpha} \mathbf{s}_{i}\right\|^{2}\right\}
$$

with respect to $i$. This operation can be speed up by calculating and storing the release of $\hat{\alpha}$ for all hypothesis in a look up table. Note that maximization of (13) represents a symbol by symbol detection if $s_{i}$ represents a symbol. For example, for detection of QPSK signal, we have four signals; each signal represents a symbol: $\mathbf{s}_{i}(t)=\sqrt{E} e^{j \theta_{i}} P(t)$; $\theta_{i} \in\left\{0, \frac{\pi}{2}, \pi, \frac{3 \pi}{2}\right\}$ where $P(t)$ is the unit amplitude pulse waveform. In this case $\alpha_{i}$ is estimated using (12) every symbol. This provides large estimation error since the number of samples per symbol is small. Another estimation algorithm which does not depend on the number of samples per symbol can be obtained if we use a Pilot symbols to estimate the channel. This algorithm is obtained by applying the least square estimation to the linear model given by (10) and results in:

$$
\hat{\alpha}=\left(\mathbf{s}^{T} \mathbf{s}\right)^{-1} \mathbf{s}^{T} \mathbf{r}
$$

where $\mathbf{s}=\left(s(1), s(2), \ldots, s\left(K_{p}\right)\right)$ and $K_{p}$ is the length of Pilot symbols. The length of the Pilot symbols can be chosen large enough to provide accurate estimate after obtaining $\hat{\alpha}$ we maximize (13) with respect to i to detect the transmitted signal.

The structure of this receiver is shown in Fig. 1. This block first calculates the estimate of the channel parameter $\alpha$ and then calculate the log-likelihood function $\Delta_{i}$ for hypothesis $i$. The output of the log-likelihood calculation blocks are entered to the unit that takes the maximum. This maximum is the estimation of the transmitted signal vector $\hat{\mathbf{s}}_{i}$.

\section{COMPUTER SIMULATIONS AND RESULTS}

In this section, the performance of the developed receiver is evaluated for the flat fading communication channel under class-A impulsive noise environment. The simulations are performed for a binary PSK signal. The parameters that are used in the simulations are as follows. The frame length is 2048 samples. The number of the 
samples per symbol is 6 . A class-A impulsive noise, with $\Gamma=1$, is simulated and added to the signal at the input of the receiver. The signal to noise ratio is defined as $S N R=10$ $\log \left(E_{b} / N_{o}\right)$ where $N_{o} / 2$ is the noise power spectral density and $E_{b}$ is the energy per bit. similar to that ones used in case of non-fading channel. It is assumed that no phase and time offsets in the carrier. However, the effects of these offsets are studied in this section.

Now, the performance of the receiver for BPSK signal is investigated. The bit error probability versus the signal to noise ratio for $A=0.01,0.1$, and 1 is shown in Fig. 2 . in this figure, we consider ideal receiver i.e. the channel is assumed to be known at the receiver. This figure shows that as signal to noise ratio increases, the bit error probability decreases. Also, the performance of the receiver depends on the impulsive index A. As $A$ decreases, the performance of the receiver deteriorates due to the increase of the noise impulsiveness. In Fig. 3, the performance comparison between the practical receiver and the ideal receiver is performed for different values of $A$. By practical receiver, we mean a receiver that assumes the channel is unknown and uses estimation algorithm to estimate it. The estimation algorithm that uses to estimate the channel is given by (14). In the ideal receiver, the channel is assumed to be known; therefore this receiver represents unrealistic case. The figure shows that there is a performance loss between the practical receiver and the ideal receiver. The reason of this performance loss is the channel parameter estimation error. This estimation error introduces an error in the log-likelihood function and causes degradation in the receiver performance. In the ideal receiver, this error does not exist and the performance of the receiver is affected only by SNR. The figure also shows that the gap in performance between the practical and the ideal receiver is large. For example, probability of error $=10^{-4}$, and for $A=0.1$ the gap in performance is about $8 \mathrm{~dB}$. This is due to the high estimation error in this algorithm because it uses only 6 samples for estimation (number of samples per bit), this is clear from Fig. 2, and 3.

The comparison of the performance of the ideal receiver, the practical receiver which equipped with the LSE algorithm and the practical receiver which equipped with the first algorithm is shown in Fig. 4. In this figure, the LSE algorithm is denoted by AL2 and the first estimate algorithm is denoted by AL1. This figure shows that the receiver equipped with the LSE algorithm has better performance than the receiver equipped with the first algorithm. For example, at $A=0.01$ and at probability of error $=10^{-4}$, the performance gain between them is about $7 \mathrm{~dB}$ while for $A=0.1$ and at the same probability of error, the gain is about $6 \mathrm{~dB}$.

Fig. 5 shows the comparison of the mean square error of estimation (MSEE) of $|\alpha|$ for the two channel estimation algorithms for $A=0.1$ and $A=0.01$. The MSEE is high at low SNR because the noise dominates the performance of the estimator. When SNR increases, the MSEE decreases. At SNR $>22 \mathrm{~dB}$, the MSEE becomes small for both values of $A$. We can also see that the MSEE in case of $A=0.1$ is smaller than that one for $A=0.01$. The convergence in case of $A=0.1$ is faster than the convergence in case of $A=0.01$. Both Fig. 5, and 6 show that the estimation algorithm requires high SNR to 
converge. Now, we use the least square estimation (LSE) algorithm given by (14) to to estimate the channel. this algorithm uses a 50 pilot samples which is found to be sufficient for good estimation. These figures show that the receiver equipped with the LSE algorithm (AL2) has better performance than the receiver equipped with the first algorithm (AL1).

The effect of the frequency offset on the performance of the receiver for binary PSK receiver over flat fading channel is shown in Fig. 7. The figure is plotted for different values of $A(0.0001,0.1$, and 1$)$, and for different values of $S N R=10 \mathrm{~dB}$. The figure shows that the receiver is able to detect the signal reliably when the frequency offset is small. When the frequency offset increases, the receiver performance degrades rapidly. Also, Fig. 7 shows that there is a range in which the effect of frequency offset can be neglected and the symbol error probability in this range is small. This range is shown in this figure for $A=1$. This range depends on the SNR (it increases as SNR increases) and it is up to $f_{0} . T_{s}=0.04$ for $S N R=10 d B$ where $T_{s}$ is the symbol duration.

The effect of the timing offset on the performance of the developed binary PSK receiver is shown in Fig. 8. The figure is plotted for different values of $A(0.0001,0.1$ and 1$)$ for $\mathrm{SNR}=10 \mathrm{~dB}$. This figure shows that the receiver is able to detect the signal reliably when the timing offset is small. When the timing offset increases, the receiver performance degrades rapidly. Also, Fig. 8 shows that there is a range in which the effect of timing offset can be neglected and the symbol error probability in this range is small. This range is shown in this figure for $A=1$. This range depends on the SNR (it increases as SNR increases) and it is up to $\mathrm{t}_{0} / \mathrm{T}_{\mathrm{s}}=0.22$ for $\mathrm{SNR}=10 \mathrm{~dB}$.

\section{CONCLUSIONS}

An optimum receiver for digitally modulated signals in additive class-A impulsive noise for flat fading channel has been presented. The performance of the receiver has been derived theoretically and validated by simulations for a binary PSK signal as a case study. It has been shown that the performance of the developed receiver depends on the noise parameter $A$. Increasing the impulsive index $A$, enhances the performance of the receiver. In fading channels, there is a performance loss between the receiver equipped with channel estimator and the receiver which assumed known channel. The reason of this performance loss is the channel estimation error.

There is a range at which the effect of frequency offset can be neglected and the receiver can detect the signal reliably. This range depends on the SNR and the impulsive index $A$. This range increases as SNR increases and A increases. When the frequency offset increases, the receiver performance degrades rapidly. The same conclusion can be stated for the timing offset. 


\section{REFERENCES}

[1] A. El-Mahdy, "Classification of NFSK Signals over Time Varying Flat Correlated Fading Channels under Class-A Impulsive Noise Environment", IEE proceedings. Commun., vol. 151, No. 6, Dec.2004, pp. 619-626.

[2] S. Kosmopoulos, M. Gouta, and P. Mathiopoulos, "Performance Evaluation of M-ary QPRS Schemes in Sever Impulsive Noise Environments", IEEE Trans. Commun., vol.39, No. 3, March 1991, pp.405-408.

[3] S. Miyamoto, M. Katayama, and N. Morinaga, "Performance Analysis of QAM Systems Under Class-A Impulsive Noise Environment", IEEE Trans. Electomagn. Compat., vol. 37, No. 2, May 1995, pp. 260-267.

[4] J. Haring, and A. Vinck, "Performance Bounds for Optimum and Suboptimum Reception Under Class-A Impulsive Noise", IEEE Trans. Commun., vol. 50, No. 7, July 2002, pp. 1130-1136.

[5] D. Middelton, "Non-Gaussian Noise Models in Signal Processing for Telecommunications: New Methods and Results for Class-A and Class-B Noise Models," IEEE Trans. Inform. Theory, vol. 45, No.4, May. 1999, pp. 11291149.

[6] Saleem A. Kassam, "Signal Detection in Non-Gaussian Noise," New York: Downden and culver, 1987.

[7] L. A. Berry, "Understanding Middleton's Canonical Formula for Class-A Noise," IEEE Trans. Commun., vol. EMC-23, no. 4, Nov. 1981.

[8] John G. Proakis, "Digital communications", McGraw-Hill Book Company.

[9] Ernesto C., Maurizio D., Maurizio L., and Marco L,"Canonical Detection in Spherically Invariant Noise," IEEE Trans. Commun., vol. 43, no. 2/3/4, Feb./March/April 1995. 


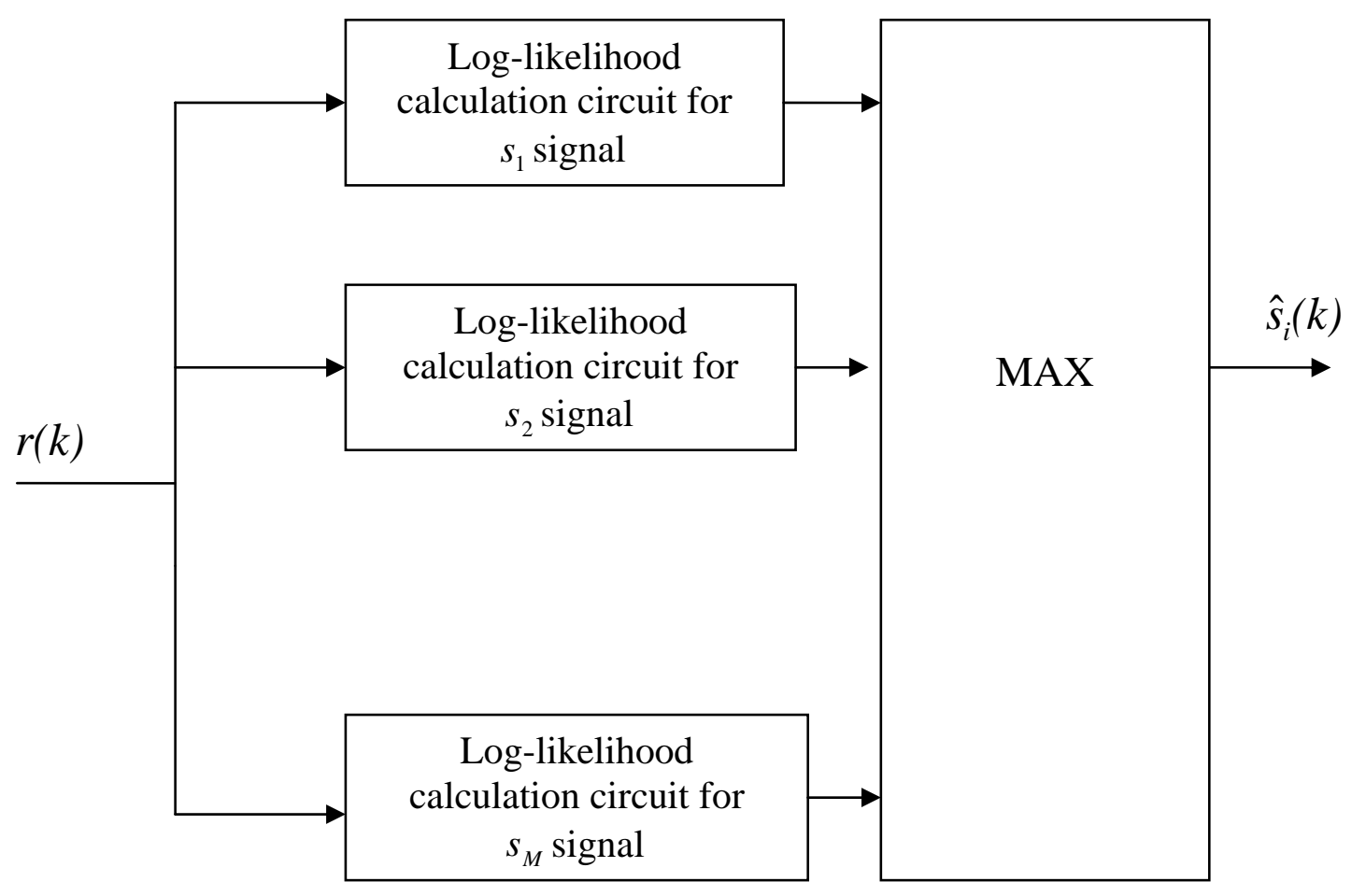

(a) Construction of the $M L$ receiver under class-A impulsive noise 

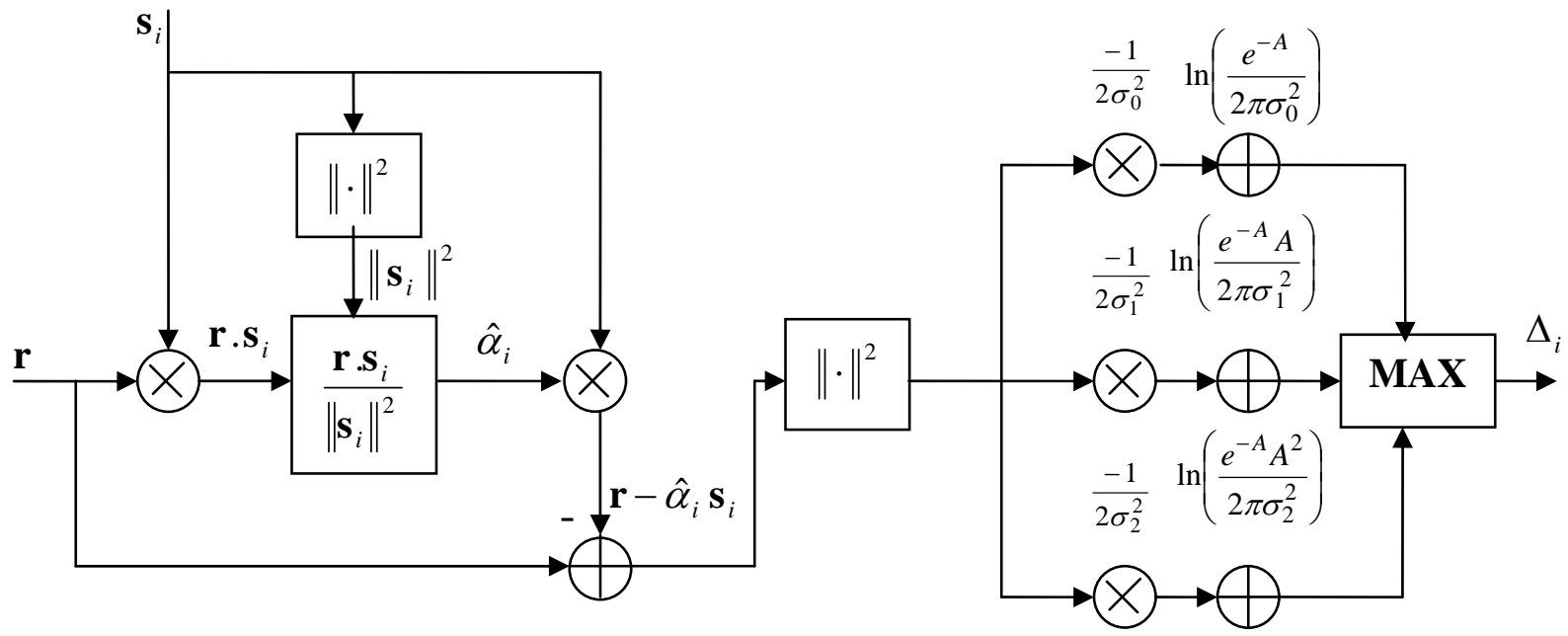

(b) Using the first estimation algorithm (eq.(12))

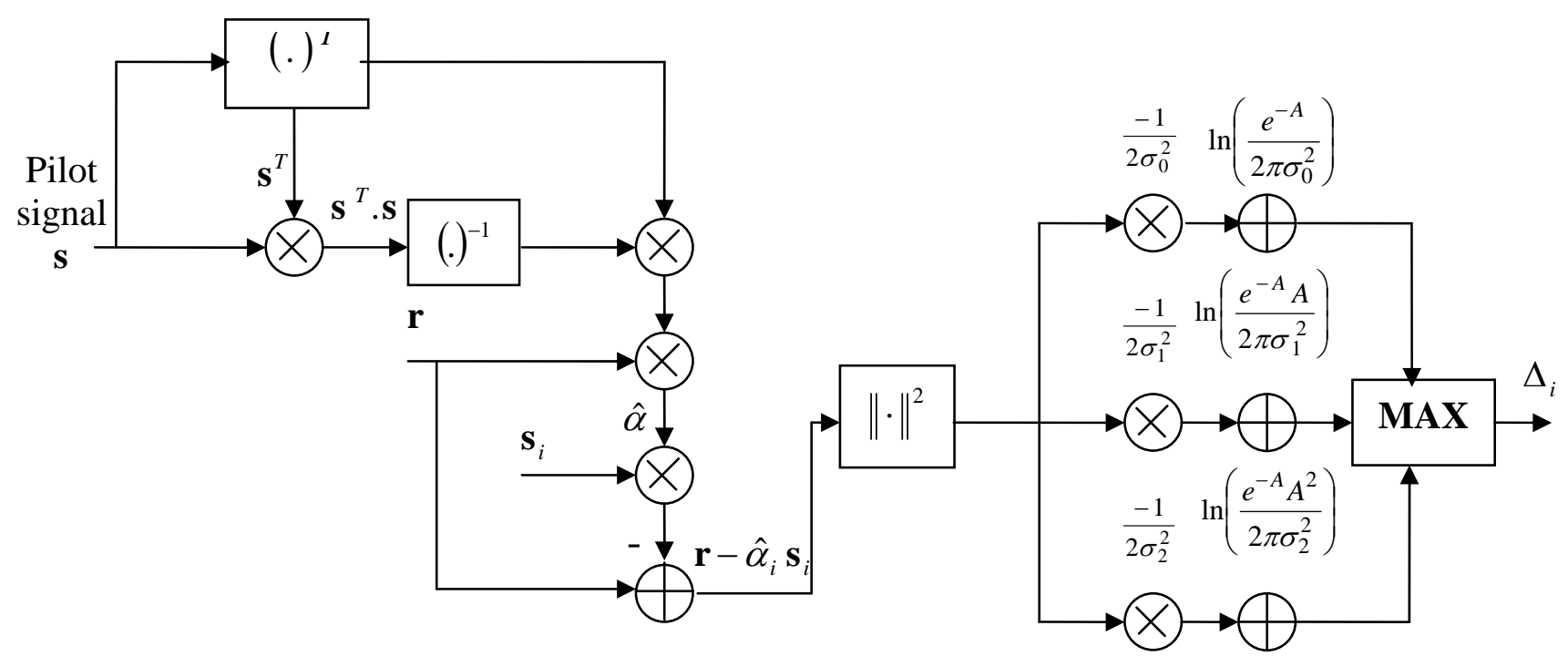

(c) Using the second estimation algorithm (eq.(14))

Fig. 1 Construction of log-likelihood calculation block for flat fading channel under classA impulsive noise 


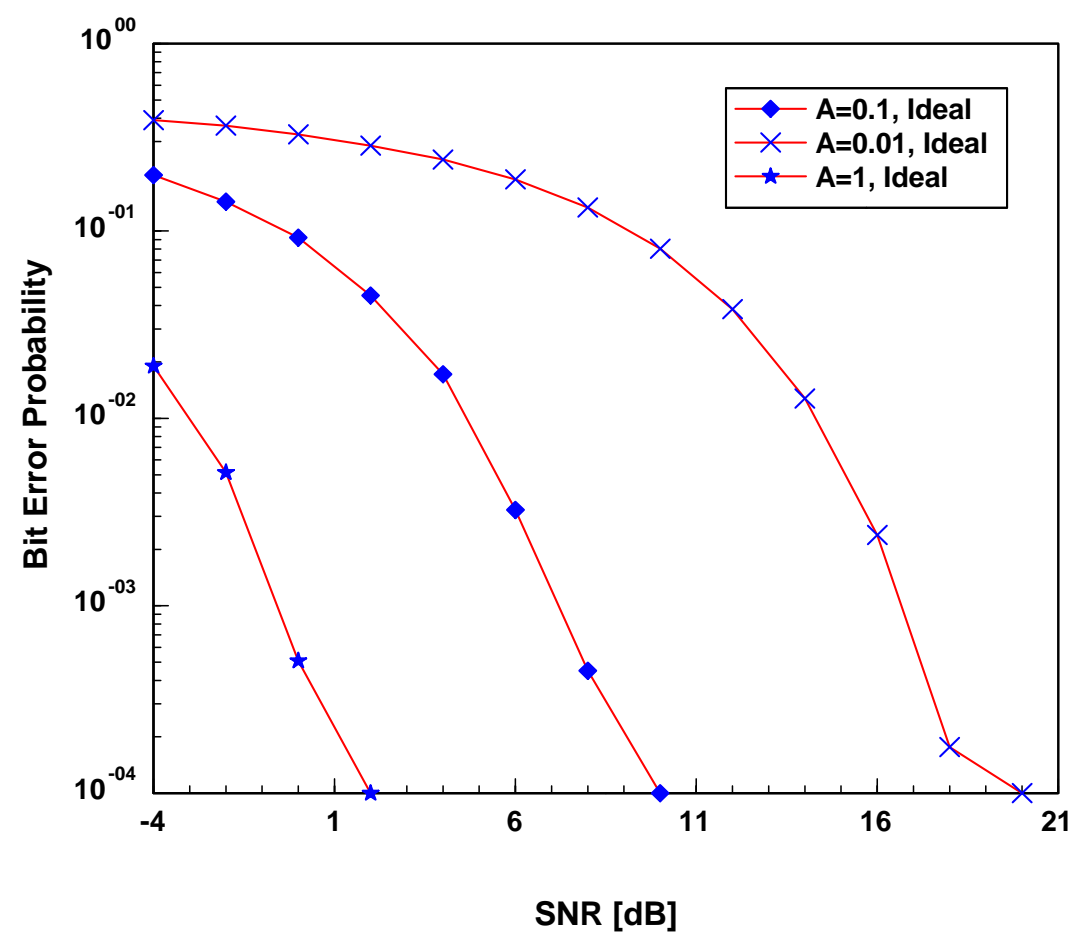

Fig. 2 Performance of the ideal receiver (known channel) for different values of the impulsive index $A$

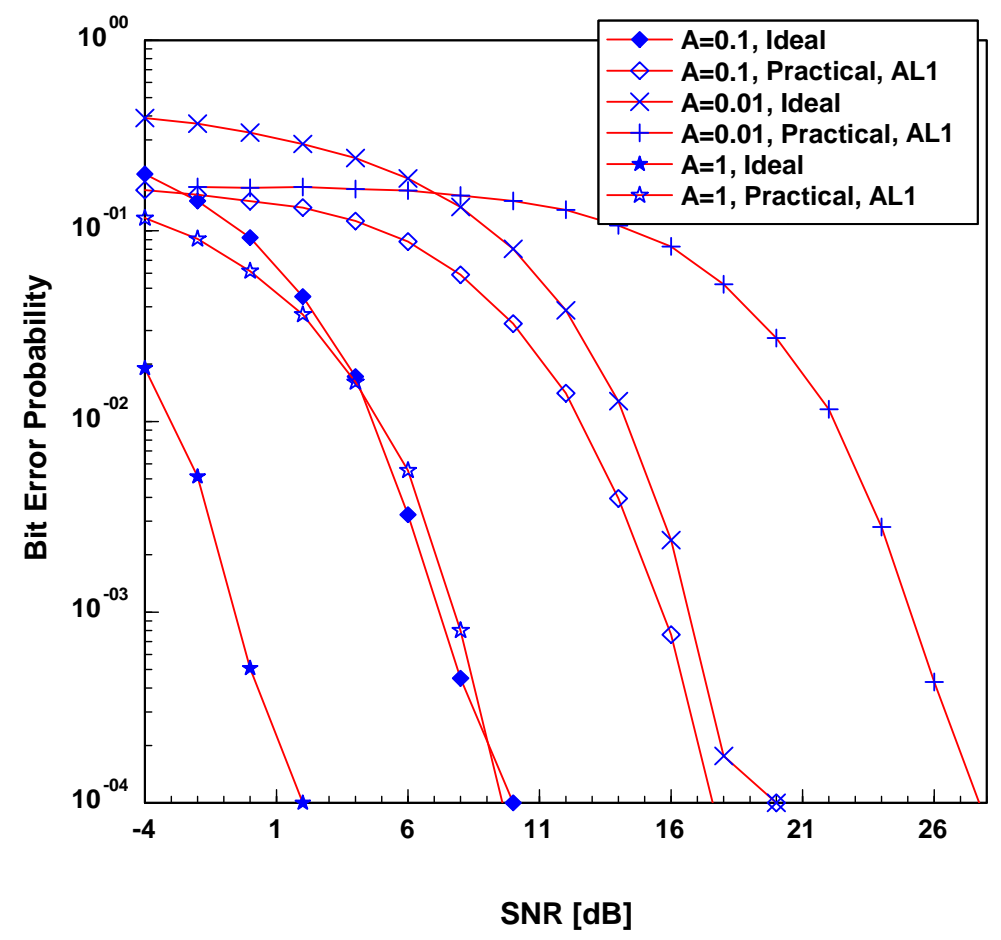

Fig. 3 Performance of the ideal and the practical receiver (estimated channel) for different values of the impulsive index $A$ 


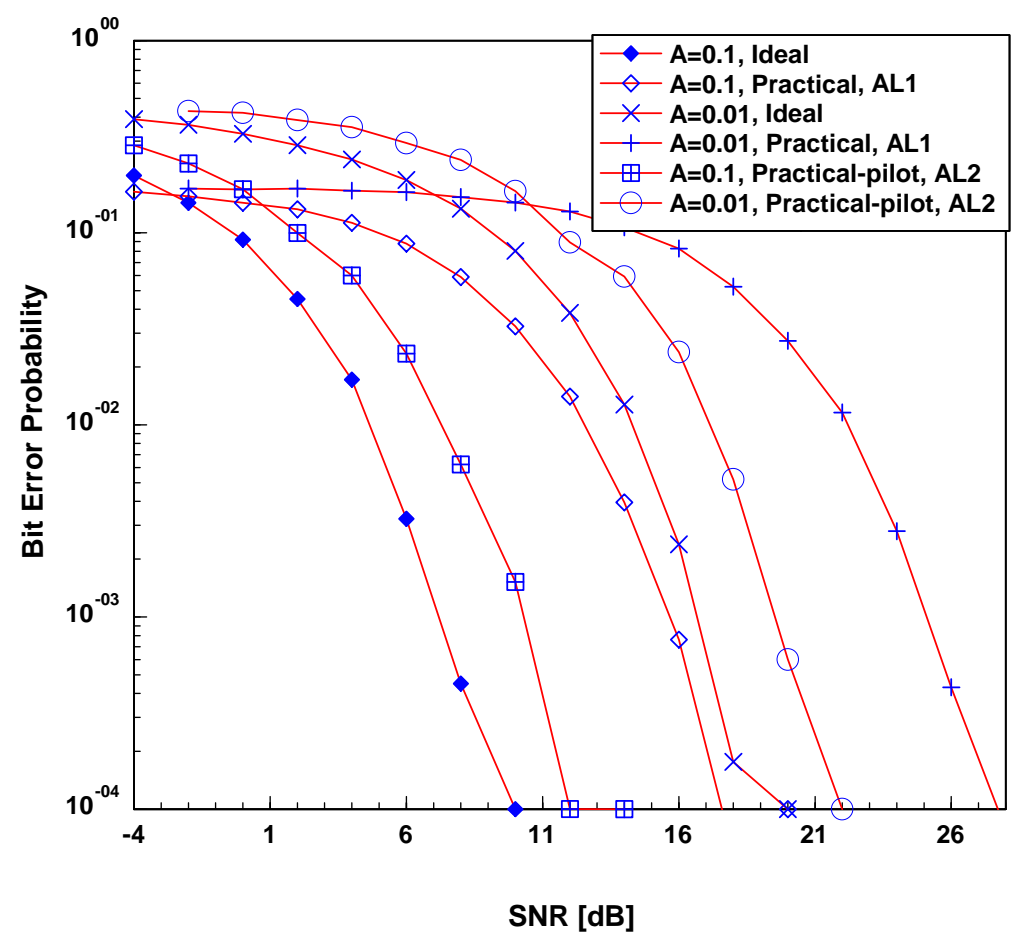

Fig. 4 Comparison of the performance of the ideal receiver, the practical receiver using the least square channel estimation algorithm (eq.(13)), and the practical receiver using the first channel estimation algorithm (eq. (14))

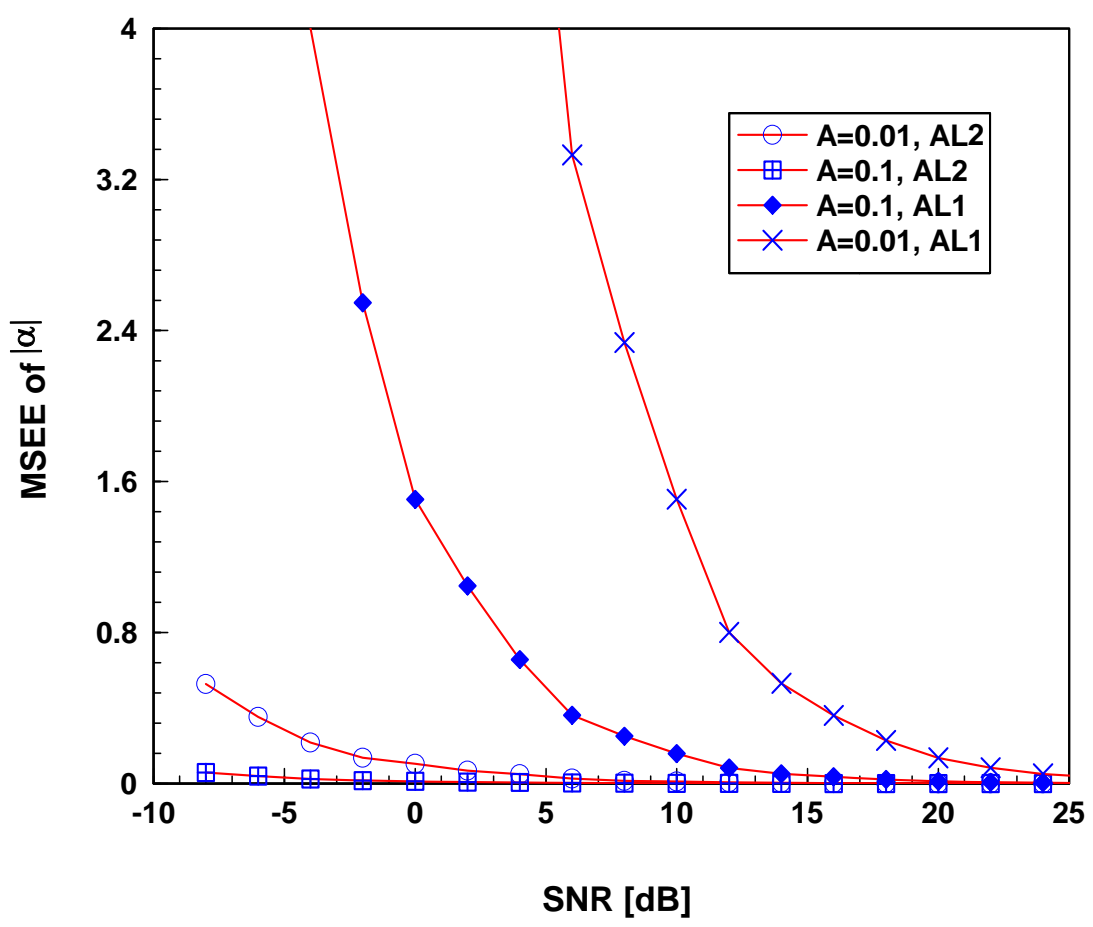


Fig. 5 Comparison of the mean square error of estimation of $|\alpha|$ for the two channel estimation algorithms for $A=0.1$ and $A=0.01$

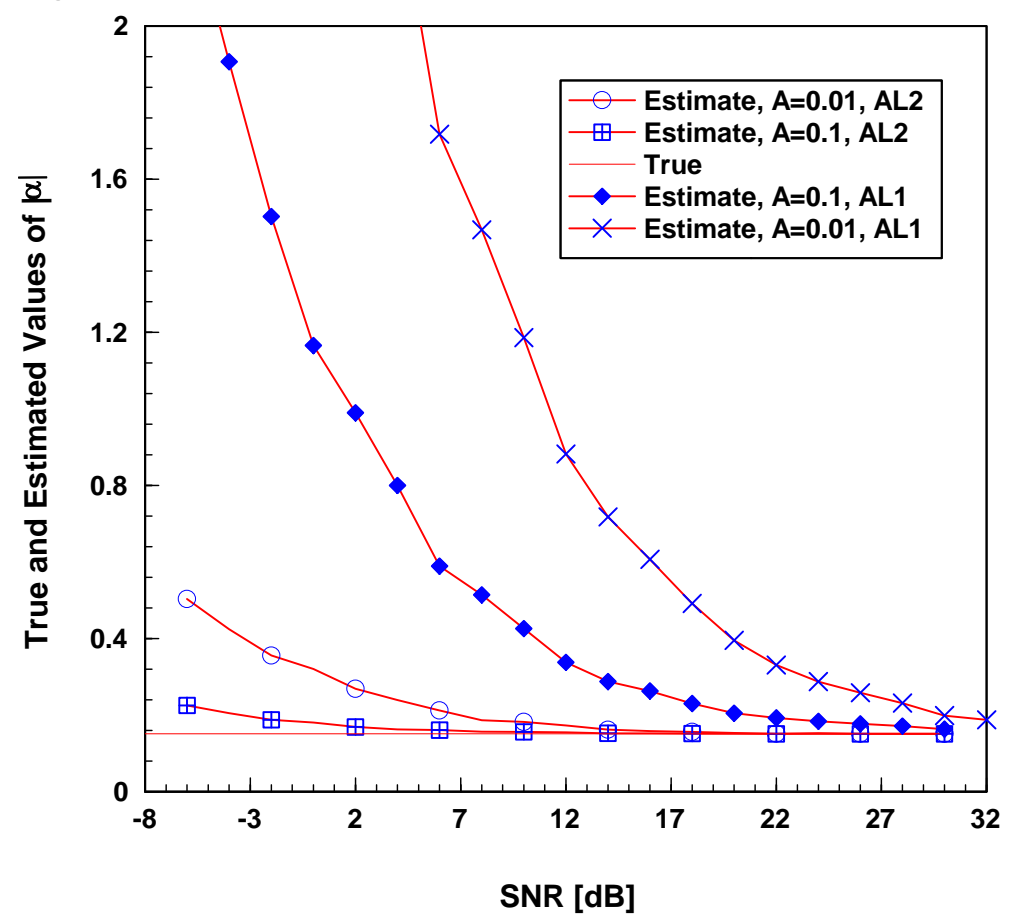

Fig. 6 Comparison of True and estimated values of fading channel coefficients $|\alpha|$ using the least square channel estimation algorithm for $A=0.1$ and $A=0.01$

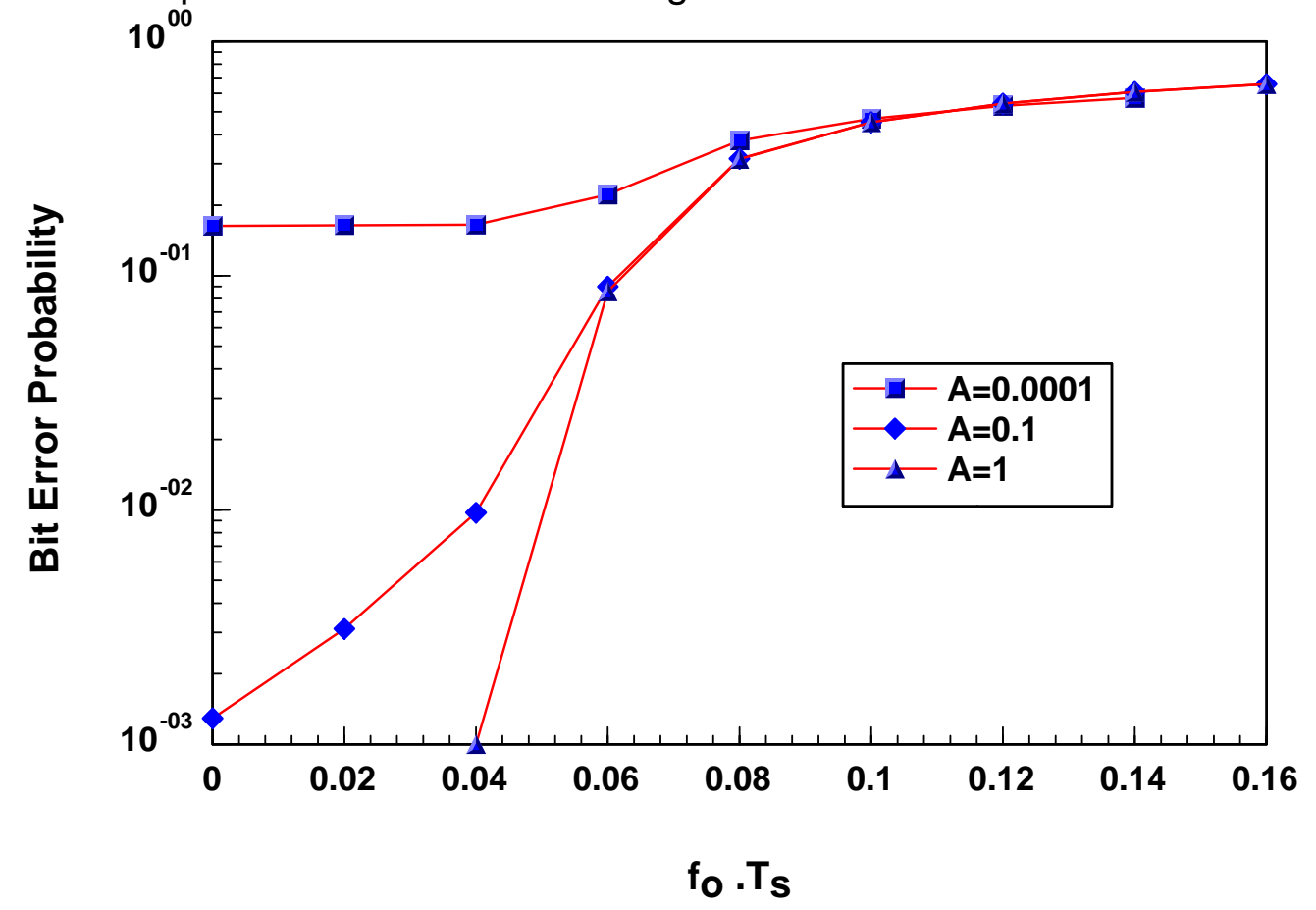


Fig. 7 Bit error probability due to frequency offset for different values of the impulsive index $A$ and for $S N R=10 \mathrm{~dB}$

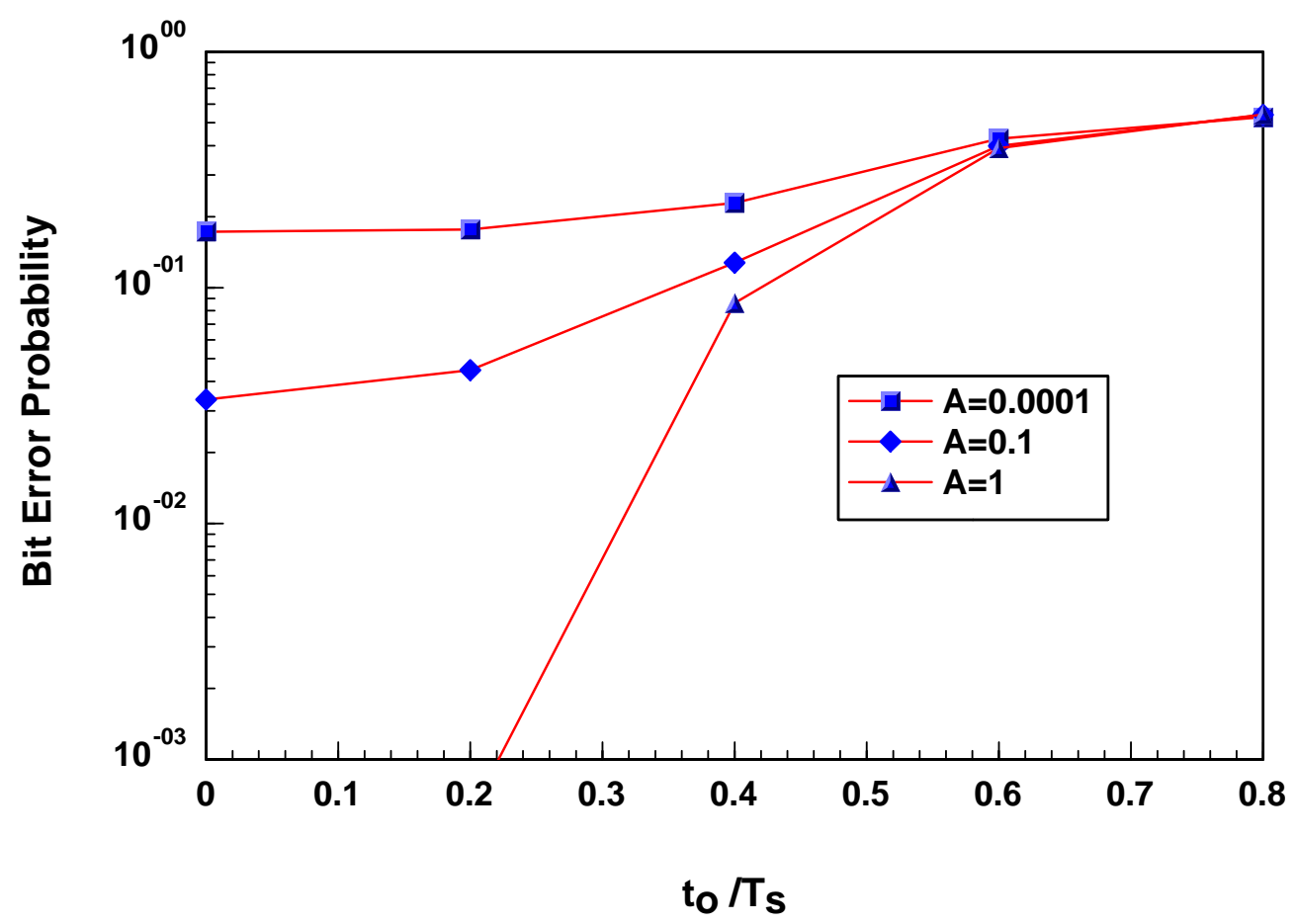

Fig. 8 Bit error probability due to time offset for different values of the impulsive index $A$ and for $\mathrm{SNR}=10 \mathrm{~dB}$ 